Biochimica et Biophysica Acta, 626 (1980) 41-56

(c) Elsevier/North-Holland Biomedical Press

BBA 38534

\title{
ANALYSIS OF INTERACTIONS AMONG PURIFIED COMPONENTS OF THE LIVER MICROSOMAL CYTOCHROME P-450-CONTAINING MONOOXYGENASE SYSTEM BY SECOND DERIVATIVE SPECTROSCOPY
}

\author{
K. RUCKPAUL ${ }^{a}$, H. REIN ${ }^{a}$, D.P. BALLOU ${ }^{b}$ and M.J. COON ${ }^{b}$ \\ a Central Institute of Molecular Biology, Department of Biocatalysis, Academy of Sciences \\ of the G.D.R., Berlin-Buch, (G.D.R.) and b Department of Biological Chemistry, The \\ University of Michigan, Ann Arbor, MI 48109 (U.S.A.)
}

(Received November 20th, 1979)

(Revised manuscript received April 11th, 1980)

Key words: Monooxygenase system; Cytochrome P-450; Derivative spectroscopy

\section{Summary}

Second derivative spectroscopy together with the respective difference spectroscopy offers an effective methodical tool to resolve overlapping bands and shoulders into distinct bands at eliminated background absorption. The improved resolution allows attribution of the distinct bands to individual amino acid residues. Both methods have been utilized to analyze interactions between the three essential components of the liver microsomal cytochrome $P$-450-containing monooxygenase system. The improved resolution of the aromatic amino acid residues in the derivative spectra of cytochrome $P-450_{\mathrm{LM} 2}$ and reductase allows one to determine that in the interactions of the essential components tyrosine residue(s) are involved. The participation of phenylalanine is likely and the participation of tryptophan residues is excluded. The $\mathrm{pH}$-dependent decrease of the tyrosine absorption bands in the medium ultraviolet region with increasing $\mathrm{pH}$ is accompanied by a concurrent decrease of the heme absorption in the Soret region. Based on this concurrence, the existence of a heme-linked tyrosine as one of the axial heme iron ligands in cytochrome $P-450$ is postulated.

\section{Introduction}

The cytochrome $P-450$-containing monooxygenase system present in the endoplasmic reticulum of mammalian liver cells brings about the hydroxylation Nomenclature: Cy tochrome $P-450_{\text {LM2 }}$ or $P-450_{\text {LM4 }}$, electrophoretically homogeneous fractions of liver
microsomal cy tochrome $P-450$. 
of a large variety of drugs and xenobiotics. The catalytic activity of the system is determined by interactions among the three essential components: cytochrome $P-450$ (the terminal oxidase), NADPH-cytochrome $P-450$ reductase and phosphatidylcholine [1]. The detailed nature of these interactions despite remarkable progress in characterization of the individual components and in elaboration of the mechanism of action of the overall system - has not been elucidated. Nothing is known about the identity of the amino acid residues involved in the binding of the components, but because of the membranous state of the system it is expected that the functional interactions are apolar in nature, involving appropriate aliphatic or aromatic amino acid residues. The aromatic amino acids are more easily studied in this respect because changes in the physiochemical environment are reflected in alterations of their spectra. The absorption spectra of proteins are characterized by absorption bands in the middle ultraviolet region composed of overlapping gaussian curves originating from $\pi-\pi^{*}$ transitions of Trp, Tyr, and Phe [2]. Unfortunately, the poor resolution of the electronic bands and shoulders makes correlation with distinct amino acid chromophores difficult, even with the aid of difference spectroscopy.

An appropriate means for high resolution of spectral bands of aromatic amino acid residues in proteins is provided by derivative spectroscopy*, which provides highly resolved and sharpened bands, thus allowing an assignment to individual amino acids [3-5].

Function-linked conformational changes of proteins usually are connected with changes of the microenvironment of amino acid residues, including aromatic residue. In the second derivative spectra, such milieu-dependent alterations are reflected in changes of the band amplitudes of the respective amino acid residues. The combination of the derivative with the difference spectroscopy offers the possibility of a sensitive qualitative attribution and a quantitative evaluation of the amplitudes and by this the determination of small conformational changes of the protein. Interpreting such changes, one may take advantage of the well known fact that a red shift of the absorption bands of the aromatic amino acids within a protein [6-9] is mainly induced by a decrease in polarity of the protein environment influencing $\pi-\pi^{*}$ transitions [2].

The better resolution of overlapping bands in the second derivative spectrum is caused by a diminution of the half-bandwidth of the negative peak of gaussian or a Lorentzian curve as compared to the basic function. The halfbandwidth of a negative peak in the second derivative of a gaussian absorption band is related to that in the basic function as $0.53: 1$ and in the case of Lorentzian bands as 0.33:1.

Furthermore, an improved resolution is achieved by the inverse quadratic dependence of the amplitude of the negative peak of the second derivative spectrum on the bandwidth parameter. Therefore, a distinct sharpening of small bands is achieved, whereas broad absorption lines as background are

\footnotetext{
* The terms derivative and derivative difference spectroscopy in the text refer to second derivative and second derivative difference spectroscopy, respectively.
} 
eliminated. For example, the relatively broad heme absorption bands in the medium ultraviolet region are eliminated in the derivative spectra [5].

The extinction curve of an absorption band can be described according to Lowry and Hudson [cf. Ref. 2] by

$A=A_{0} \exp \left(-\frac{\left(\nu-\nu_{0}\right)^{2} \nu_{0}^{2}}{\gamma_{0}^{2} \nu^{2}}\right)$

where $\nu_{0}$ is the position of the peak in wavenumbers $\left(\mathrm{cm}^{-1}\right), \nu$ is the position, in wavenumbers, at which the extinction $(A)$ is being determined, and $\gamma_{0}$ is the band width in wavenumbers. Since $\nu=1 / \lambda$,

$A=A_{0} \exp \left(-\frac{\left(\lambda-\lambda_{0}\right)^{2}}{\lambda_{0}{ }^{4} \gamma_{0}{ }^{2}}\right)=A_{0} \exp \left(-\frac{\left(\lambda-\lambda_{0}\right)^{2}}{\Gamma_{0}{ }^{2}}\right)$

The second derivative of this equation is

$\frac{\mathrm{d}^{2} A}{\mathrm{~d} \lambda^{2}}=\frac{2 A_{0}}{\Gamma^{2}}\left(\frac{2\left(\lambda-\lambda_{0}\right)^{2}}{\Gamma^{2}}-1\right) \exp \left(-\frac{\left(\lambda-\lambda_{0}\right)^{2}}{\Gamma^{2}}\right)$

where $A_{0}=\epsilon \cdot c \cdot d$; and $\gamma, \Gamma=$ bandwidth parameter in wavelength. The amplitude of the signal in the second derivative spectrum is directly proportional to the concentration of the chromophore. The half-bandwidth of the function in Eqn. 1 is:

$\Delta \lambda_{2 / 2}=2 \sqrt{\ln 2} \Gamma=1.665 \Gamma$

The half-bandwidth of the second derivative (negative peak) from Eqn. 2 is:

$\Delta \lambda_{1 / 2}=0.885 \Gamma$

The amplitude of the negative peak in the second derivative spectrum relative to the baseline is:

Amplitude $=2 A_{0} / \Gamma^{2}$

The peak-to-trough difference, however is

Amplitude (peaktrough) $=2.89 A_{0} / \Gamma^{2}$

In the present paper the mutual influences of the components of the enzyme system are studied using second derivative and second derivative difference spectroscopy. From the data obtained, it appears that tyrosine is involved in these interactions. Studies on the $\mathrm{pH}$ dependence showed that the spectral change observed upon interaction with cytochrome $P-450_{\mathrm{LM} 2}$ could be reproduced by decreasing the degree of ionization of tyrosine. The results may possibly indicate that the sixth ligand to the heme iron is a tyrosine residue and that phospholipid may help regulate the interaction between the cytochrome and the reductase. 


\section{Materials and Methods}

The spectroscopy measurements were carried out using a dual wavelength UV-300 spectrophotometer (Shimadzu, Kyoto, Japan) with the DES-1 derivative spectrophotometric accessory unit. Standard conditions for the measurement of second derivative spectra were: scan speed $75 \mathrm{~nm} / \mathrm{min}$ resulting in a $\Delta \lambda$ of $2 \mathrm{~nm}$ (time response $=0.05 \mathrm{~s}$ ). Higher resolution for the titration experiments with liver microsomal cytochrome $P-450$ was achieved by increasing the scan speed to $150 \mathrm{~nm} / \mathrm{min}$, resulting in a $\Delta \lambda$ of $4 \mathrm{~nm}$ (time response $=0.2 \mathrm{~s}$ ). $\mathrm{pH}$ measurements were performed by means of a $\mathrm{pH}$ meter MV 85 (Clamann und Grahnert, Dresden, G.D.R.).

Cytochrome $P-450_{\mathrm{LM} 2}$ and NADPH-cytochrome $P-450$ reductase were isolated in electrophoretically homogeneous form from liver microsomes of phenobarbital-induced rabbits according to published procedures $[10,11]$. The cytochrome preparation had a specific content of $12 \mathrm{nmol}$ per mg protein, and the reductase preparation had a specific activity of $44 \mathrm{nmol}$ of cytochrome $c$ reduced at $30^{\circ} \mathrm{C}$ per $\mathrm{mg}$ of protein per min. Dimyristoyl phosphatidylcholine was prepared from egg yolk [12] and subsequently dissolved in a 1:1 benzene/ methanol mixture. The solvent was removed by evaporation, and the dry dimyristoyl phosphatidylcholine was dispersed in a buffer solution $(0.1 \mathrm{M}$ phosphate buffer, $\mathrm{pH}$ 7.4) by sonication and used in this form for reconstitution experiments.

\section{Results}

\section{Second derivative spectra}

In Figs. 1 and 2 the absorption spectra together with the corresponding derivative spectra of cytochrome $P-450_{\mathrm{LM} 2}$ and of cytochrome $P-450$ reductase are given. The comparison of both types of spectra clearly indicates the far higher resolution of the transitions due to the aromatic amino acids as measured by the second derivative as compared to the absorption spectra. The derivative spectra of the single aromatic amino acids Trp, Tyr and Phe in aqueous buffered solution as well as of a mixture corresponding to the aromatic amino acid composition of the proteins investigated were recorded in order to permit attribution of the bands to the respective amino acids.

From Table I it is seen that Trp and Tyr overlap with bands at $279.8 \mathrm{~nm}$ and $280.5 \mathrm{~nm}$, respectively, whereas the Phe bands at shorter wavelengths and the Trp band at $286.5 \mathrm{~nm}$ show no overlapping with other bands. For the evaluation of the different amino acids in the proteins, therefore, the characteristic minimum at 286.5 for $\operatorname{Trp}\left(290.2 \mathrm{~nm}\right.$ in cytochrome $\left.P-450_{\mathrm{LM} 2}\right)$ and at 255.8 $\mathrm{nm}$ for Phe $\left(257.2 \mathrm{~nm}\right.$ in cytochrome $\left.P-450_{\mathrm{LM} 2}\right)$ were used. For the quantitation of Tyr the main band at $280.5 \mathrm{~nm}\left(283 \mathrm{~nm}\right.$ in cytochrome $\left.P-450_{\mathrm{LM}}\right)$ was used, despite overlapping with the Trp band. Due to the composition of cytochrome $P-450_{\mathrm{LM} 2}$ (nine Tyr and one Trp) the contribution of Trp is minimal and therefore this band is mainly determined by Tyr. The spectra of the amino acid mixtures corresponding to the proteins show almost identical positions of the bands as compared to those of the single amino acids. Moreover, the simulated spectra also show great similarity with those of the proteins. In Figs. 


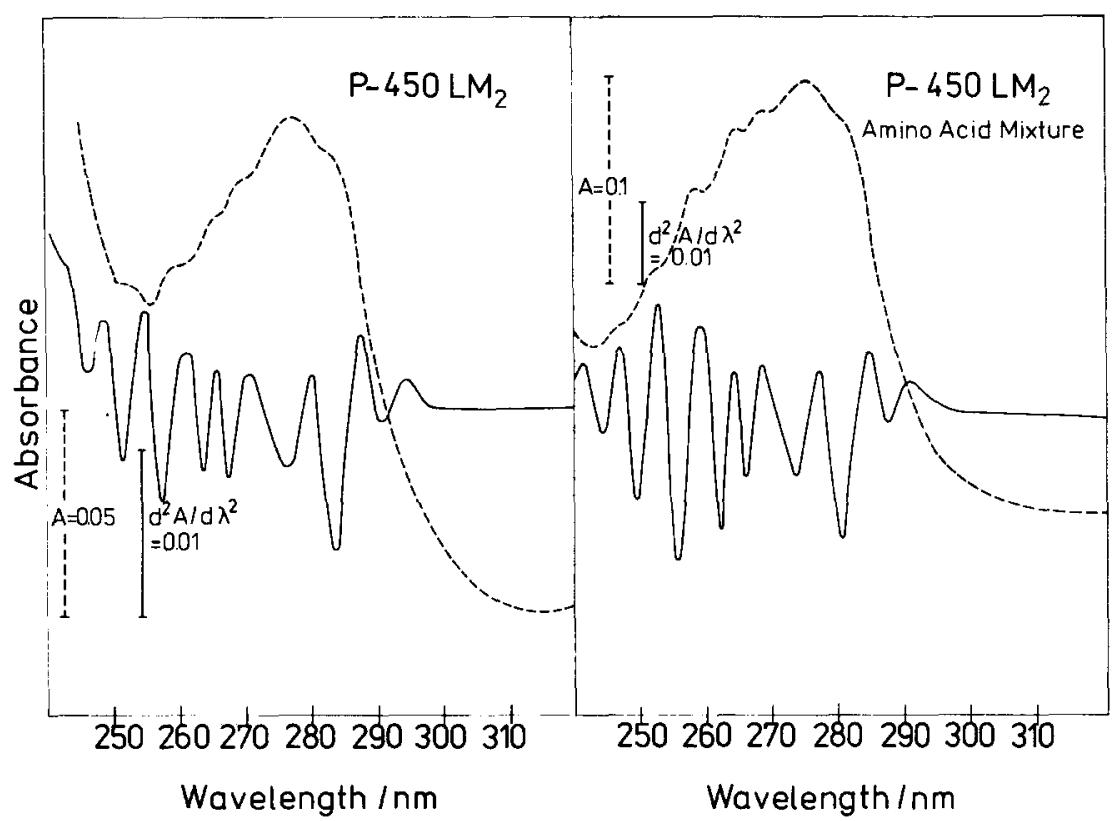

Fig. 1. Comparison of the absorption spectrum and the second derivative spectrum of liver microsomal cytochrome $P \mathbf{P 5 0}$ with the respective spectra of the corresponding amino acid mixture in the spectral region 240-320 nm. Experimental conditions: cytochrome $P-450_{\mathrm{LM} 2}=3.3 \mu \mathrm{M} ; 0.1 \mathrm{M}$ phosphate buffer, pH 7.4; the concentration of Trp in the mixture analogous to cytochrome $P-450_{L M 2}$ was adjusted to $1 \cdot 10^{-5} \mathrm{M}$. The concentrations of the other amino acids were related to the concentration of Trp according to the amino acid composition: Trp, 1; Tyr, 9; Phe, 31. The mixture was dissolved in the same buffer solution as the protein. Light path, $1 \mathrm{~cm}$; room temperature.

1 and 2 as well as in Table I a red shift* of the minima of the bands in the derivative spectra can be seen: $2.5-2.7 \mathrm{~nm}$ for Tyr and Trp and 1.5-1.7 nm for Phe. This red shift of the chromophores of the aromatic amino acids in proteins is well known [9], and explicable by a shielding of water from the apolar amino acid residues in the interior of the proteins.

\section{Second derivative difference spectra}

The second derivative difference spectra were recorded in order to analyse the interactions among the essential components of the liver microsomal monooxygenatic system. Buffered solutions of the isolated, purified components were put into tandem cuvettes. The spectrum of the mixture of components in the sample cuvette was measured against that of separated components in the reference cuvette.

In Fig. 3 the derivative difference spectra are shown, indicating the interactions of cytochrome $P-450_{\mathrm{LM} 2}$ with dimyristoyl phosphatidylcholine (Fig. $3 a)$, with cytochrome $P-450$ reductase (Fig. $3 b$ ) and in the complete reconstituted system (Fig. 3c).

\footnotetext{
* Red shift or blue shift in the text denotes irrespective of the spectral region a shift to longer and to shorter wavelengths, respectively.
} 


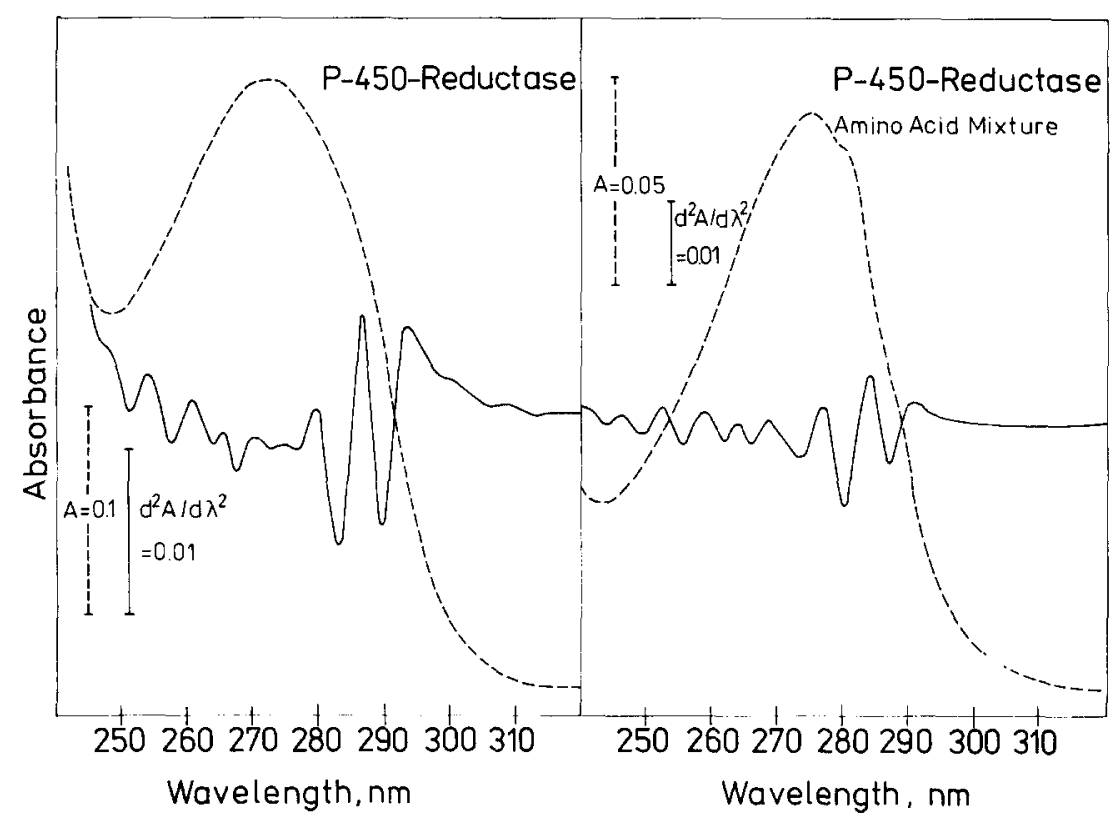

Fig. 2. Comparison of the absorption spectrum and the second derivative spectrum of NADPH-cytochrome $P-450$ reductase with the respective spectra of the corresponding amino acid mix ture in the spectral region 240-320 nm. Experimental conditions: reductase $=2.2 \mu \mathrm{M} ; 0.1 \mathrm{M}$ phosphate buffer, $\mathrm{pH} 7.4$, containing $1 \cdot 10^{-4} \mathrm{M}$ EDTA; the concentration of Trp in the mixture analogous to the reductase was adjusted to $1 \cdot 10^{-5} \mathrm{M}$. The concentrations of the other amino acids were related to the concentration of Trp according to the amino acid composition: Trp, 6; Tyr, 28; Phe, 25. The mixture was dissolved in the same buffer solution as the protein. Light path, $1 \mathrm{~cm}$; room temperature.

The baseline shown in all figures corresponds to the derivative difference spectra before components were mixed in the sample cuvette. The interaction between cytochrome $P-450_{\mathrm{LM} 2}$ and phosphatidylcholine (Fig. 3a) leads to minima at $282.5 \mathrm{~nm}$ and $275.0 \mathrm{~nm}$, clearly indicating the characteristic minima of the Tyr bands in the proteins. This shows that in the presence of phospholipid the amplitudes of the Tyr bands increase. The same results were obtained in analogous experiments with dilauroyl phosphatidylcholine.

In other experiments not presented, it was shown that phospholipid does not exert similar effects on the aromatic amino acids of the reductase. Obviously, the effect of phospholipid on Tyr and Phe is specific for cytochrome $P-450_{\mathrm{LM} 2}$. The interaction of reductase with cytochrome $P-450_{\mathrm{LM} 2}$ shows an effect opposite to that of phospholipid; the bands at $275 \mathrm{~nm}$ and $282 \mathrm{~nm}$ attributable to Tyr now are positive. Thus, the amplitudes of the Tyr bands in the derivative difference spectrum due to interaction of reductase with cytochrome $P-450_{\mathrm{LM} 2}$ diminish. We assume that this decrease is due to a Tyr residue(s) in cytochrome $P-450_{\mathrm{LM} 2}$ rather than in reductase as will be discussed later.

Fig. 3c shows the derivative difference spectrum due to interaction of all three components in a reconstituted system. Since the mixture of reductase and phospholipid showed no effect, these two components were put into one compartment and cytochrome $P-450_{\mathrm{LM} 2}$ into the other compartment of the tandem cuvette. In this spectrum, just as in that in Fig. 3a, band positions as 


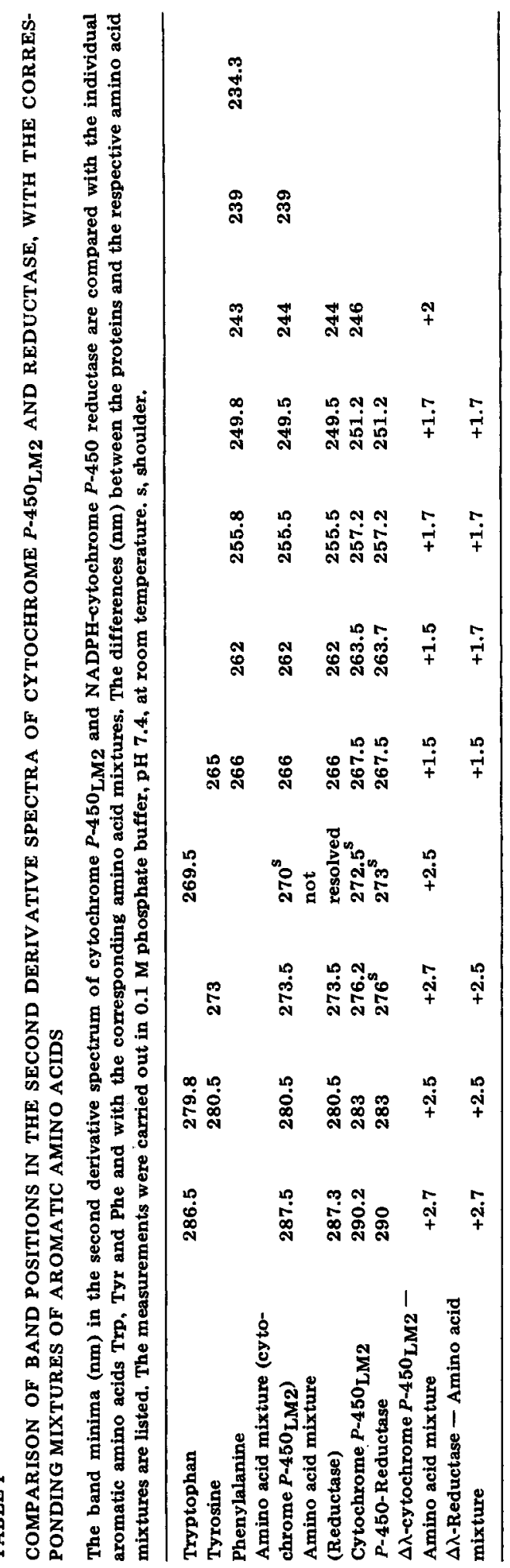




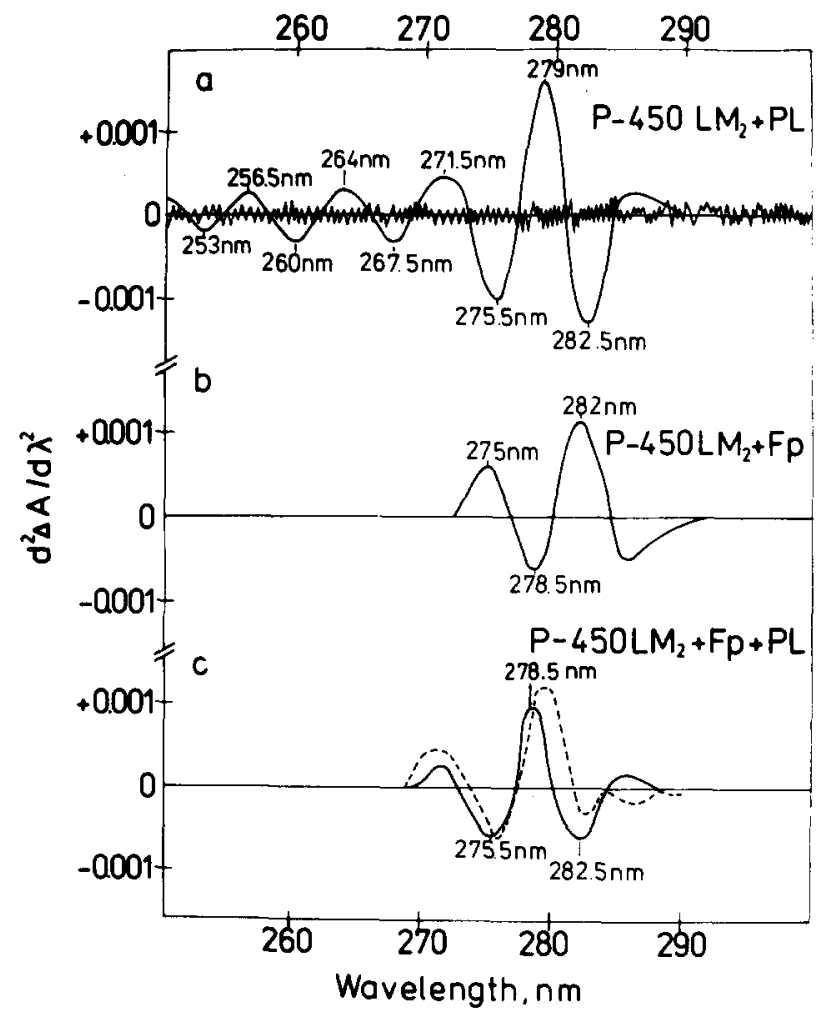

Fig. 3. Component interactions as reflected in the second derivative difference spectra in the spectral region 250-300 nm. For all measurements, tandem cuvettes were used. The baseline corresponds to the spectral properties before mixing. The concentrations given are those obtained after the reactants were mixed.

(a) Interaction between cytochrome $P-450$ and dimyristoyl phosphatidylcholine (PL). Experimental conditions: cytochrome $P-450$ LM2 $=3.3 \mu \mathrm{M} ;$ [dimyristoyl phosphatidylcholine] $=0.5 \mathrm{mM} ;$ molar ratio cytochrome $P-450_{\mathrm{LM}} /$ phospholipid $=1: 150 ; 0.1 \mathrm{M}$ phosphate buffer, $\mathrm{pH} 7.4 ;$ room temperature; tandem cuvettes: $1 \mathrm{~cm}$.

(b) Interaction between cytochrome $P-450_{\mathrm{LM}}$ and NADPH-cy tochrome $P-450$ reductase (Fp). Experimental conditions: [cytochrome $P-450_{\mathrm{LM} 2}$ ] $=3.3 \mu \mathrm{M}$; [reductase] $=2.2 \mu \mathrm{M}$; molar ratio: cy tochrome $P-450_{\text {LM2 }} /$ reductase $=1.5: 1 ; 0.1 \mathrm{M}$ phosphate buffer, $\mathrm{pH}$ 7.4; room temperature; tandem cuvettes: $1 \mathrm{~cm}$.

(c) Reconstituted system (solid line) and the calculated curve for the reconstituted system obtained by subtraction of the curve in Fig. 3b from that in Fig. 3a (dashed line). Experimental conditions: [cytochrome $P-450 \mathrm{LM}$ ] $=3.3 \mu \mathrm{M} ;$ reductase $=2.2 \mu \mathrm{M} ;$ [dimyristoyl phosphatidylcholine] $=250 \mu \mathrm{M} ; 0.1 \mathrm{M}$ phosphate buffer, pH 7.4; room temperature; tandem cuvettes: $1 \mathrm{~cm}$.

minima are formed characteristic for Tyr, indicating that in the reconstituted system the amplitudes of the Tyr band are increased.

In order to test whether additional effects are produced by the interactions of all three components as compared to the mixtures of only two components, the curve in Fig. 3b (due to cytochrome plus reductase) was subtracted from that in Fig. 3a (due to cytochrome plus phospholipid) and compared with the calculated one (dashed line in Fig. 3d). Both curves are quite similar and suggest that significant additional effects appearing in the reconstituted system can be excluded. The Tyr bands seen with the reconstituted system can be 
explained by an overlapping of interactions of cytochrome $P-450_{\mathrm{LM} 2}$ with phospholipid and with reductase.

\section{Influence of environmental changes on the spectral properties of aromatic amino acids}

(a) Solvent effects. Studies of the effect of solvents of different polarity (methanol, ethanol, $n$-propanol, isopropanol, dimethyl-formamide, dimethylsulfoxide and acetonitrile) have shown that the position of the peaks of the aromatic bands as well as amplitudes in the derivative spectra are dependent on both the nature of the nonaqueous solvent and on its concentration. The specific influences of different solvents on the $\pi-\pi *$ transitions as reflected in the derivative spectrum are under study. Quantitative analyses were carried out with ethanol [16]. With increasing amounts of ethanol (up to $50 \% \mathrm{v} / \mathrm{v}$ ) the amplitude of the spectral bands of all three amino acids increases up to 20-25\%. Concomitant with an increase in amplitudes, a red shift of about 1 $\mathrm{nm}$ is observed in buffer solution containing $50 \%$ ethanol $(\mathrm{v} / \mathrm{v})$.

(b) Analysis of the $p H$-dependence. Of the aromatic amino acids investigated, only the Tyr residue with its aromatic hydroxyl group is ionizable in the polypeptide chain. In order to prove the influence of ionization on the derivative spectrum, the $\mathrm{pH}$-dependence of the amplitude of the Tyr band at 280.5 $\mathrm{nm}$ was studied. With increasing ionization of Tyr in the $\mathrm{pH}$ range between $\mathrm{pH} 8.5$ to $\mathrm{pH}>12$ the amplitude decreases. From the titration curves, a $\mathrm{p} K$ of $9.83(0.05 \mathrm{M}$ phosphate buffer) was determined which is similar to a reported value of 10.07 calculated from a $K_{\mathrm{i}}$ of $8.5 \cdot 10^{-11}$ [13]*.

In order to obtain evidence whether the changes in amplitude of the Tyr band observed in the derivative difference spectra are caused by changes in microenvironmental effects (polarity) or changes in the degree of ionization, the derivative spectrum of cytochrome $P-450_{\mathrm{LM} 2}$ was examined for dependence on $\mathrm{pH}$ in the range from 7.5 to 12.2 together with the difference spectra in the Soret region.

With increasing $\mathrm{pH}$, similar to the results with the amino acid mixture, a decrease of the Tyr band was observed in cytochrome $P-450_{\mathrm{LM} 2}$ (Fig. 4). For quantitation of the titration the decreasing Tyr band at $283 \mathrm{~nm}$ was related to the isoclinic point at $285 \mathrm{~nm}$. The decrease of the Tyr band is paralleled by an increase of the Trp band at $290 \mathrm{~nm}$. For quantitative determination the difference of the amplitudes between $290 \mathrm{~nm}$ (minimum) and $294 \mathrm{~nm}$ (maximum) were used. The $\mathrm{pH}$ titration of the Tyr proceeds discontinuously with the formation of a step. From this behavior, two values, $\mathrm{p} K_{1}=9.9$ and $\mathrm{p} K_{2}$ $=11.35$, were obtained.

In order to prove if the ionization of Tyr is a heme-linked process in the next experiment the $\mathrm{pH}$-dependence of the heme chromophore was analyzed in the Soret region. The difference spectrum of cytochrome $P-450_{\mathrm{LM} 2}$ in the Soret region was recorded with increasing $\mathrm{pH}$ using a solution of cytochrome $P .450_{\mathrm{LM} 2}$ at the same concentration as in the reference, as shown in Fig. 5.

With increasing $\mathrm{pH}$, a difference spectrum is seen in the Soret region with a

* The difference between the $\mathrm{p} K$ described in the literature and our value could be due to the presence of more concentrated buffer in the present experiments. 


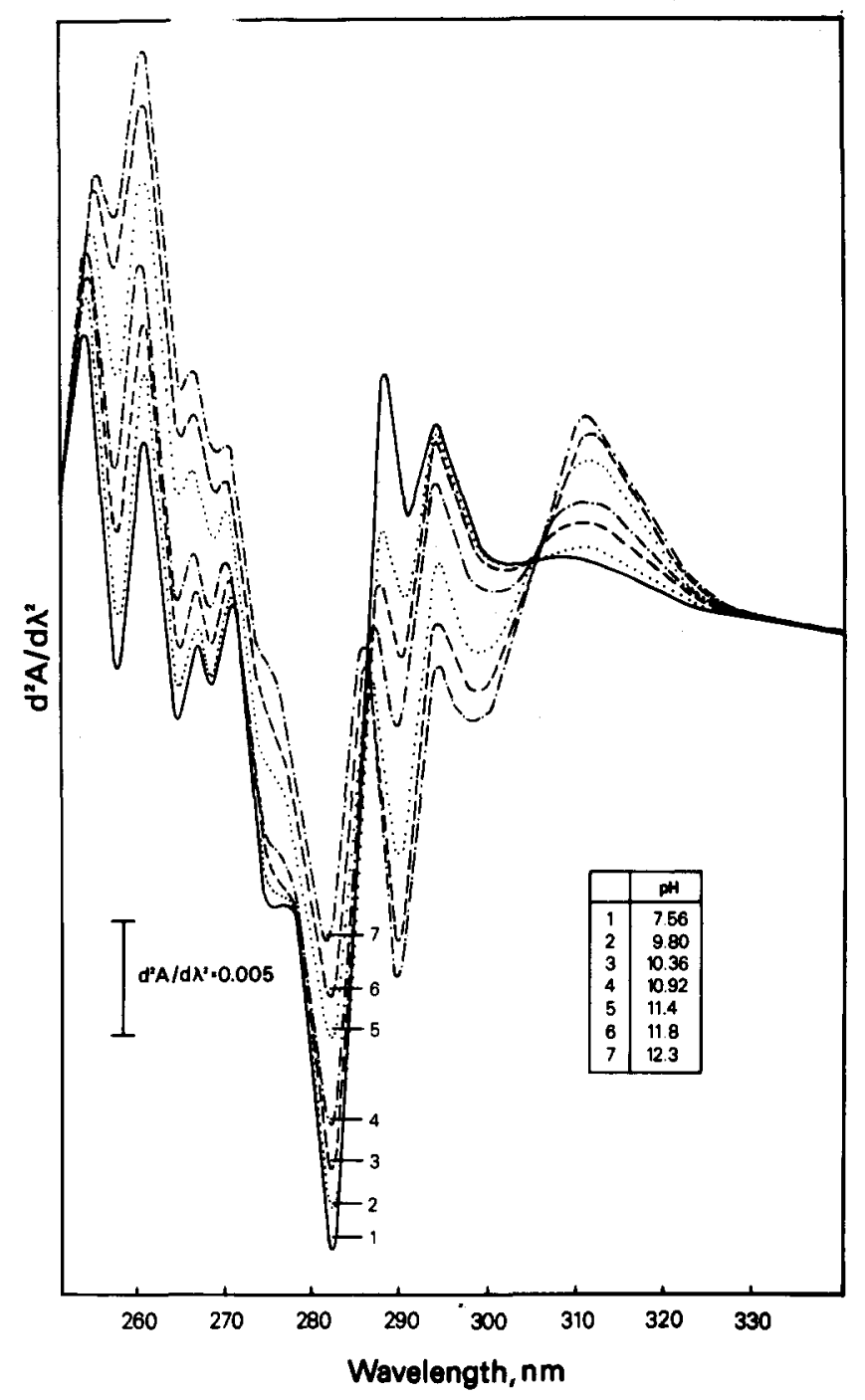

Fig. 4. Dependence on $\mathrm{pH}$ of the derivative difference spectra of cytochrome $P-450$ in the region from 250 to $340 \mathrm{~nm}$. Experimental conditions: [cy tochrome $P-450_{\mathrm{LM}}$ ] $=3.3 \mu \mathrm{M} ; 0.05 \mathrm{M}$ phosphate buffer; $10 \%$ glycerol $(\mathrm{v} / \mathrm{v})$. The $\mathrm{pH}$ was adjusted to different $\mathrm{pH}$ values in the range between 7.56 and $\mathrm{pH}>12 \mathrm{by}$ the addition of different amounts of $0.1 \mathrm{M} \mathrm{NaOH}$ up to a maximal volume of $100 \mu \mathrm{l}$ to a total volume of $2.5 \mathrm{ml}$ in the sample cuvette. The dilution of $4 \%$ caused by the added NaOH was neglected. Light path, $1 \mathrm{~cm}$.

minimum at $416 \mathrm{~nm}$ which is shifted to shorter wàvelength $(412.5 \mathrm{~nm})$ at $\mathrm{pH}$ 10.22 .

The appearance of the increasingly negative Soret band is accompanied by the formation of two isosbestic points, at $425.5 \mathrm{~nm}$ and $381.5 \mathrm{~nm}$. Above $\mathrm{pH}$ 10.22 a further increase of the amplitude of the negative band is observed which is paralleled by a back shift of the minimum to $416 \mathrm{~nm}$ at $\mathrm{pH}>12.3$ and accompanied by the disappearance of an isosbestic point. It is well known that alkalization of cytochrome $P-450_{\text {LM2 }}$ results in the formation of cyto- 


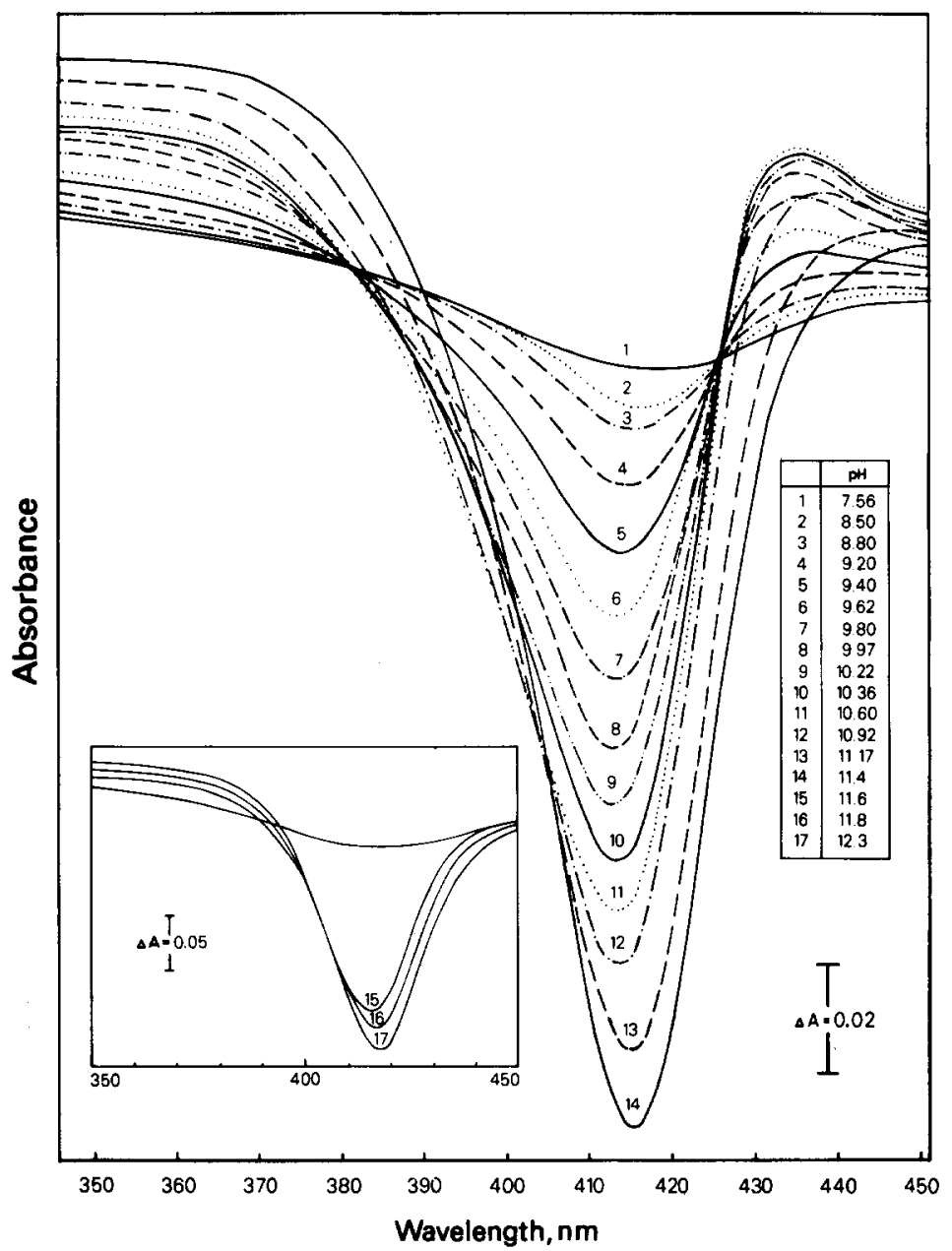

Fig. 5. Dependence on $\mathrm{pH}$ of the difference spectra of cytochrome $P-450$ in the Soret region. Experimental conditions: [cytochrome $P-450$ LM2] $=3.3 \mu \mathrm{M}: 0.05 \mathrm{M}$ phosphate buffer; $10 \%$ glycerol (v/v). The titration was performed by addition of $1 \mathrm{M} \mathrm{NaOH}$ up to a maximal volume of $100 \mu l$ to a total volume of $2.5 \mathrm{ml}$ in the sample cuvette. Spectral changes were measured against a solution of cytochrome $P$ $450_{\mathrm{LM} 2}$ at the same protein concentration at $\mathrm{pH} 7.56$ in the reference cuvette. The dilution of $4 \%$ caused by the added $\mathrm{NaOH}$ was neglected. Light path, $1 \mathrm{~cm}$.

chrome $P-420$ [22]. The determination of cytochrome $P-450$ formation revealed that the transition from cytochrome $P-450$ to cytochrome $P-420$ is accomplished at about $\mathrm{pH}$ 10. The significant red shift of the Soret band in the difference spectra (Fig. 5) at $\mathrm{pH}>10$ suggests that above this $\mathrm{pH}$ by an exchange of the axial ligand(s) a new stable heme complex is formed. This is supported by electron spin resonance measurements of liver microsomes at alkaline $\mathrm{pH}$. Under this condition the $g$-values of cytochrome $P-450$ are 1.87 , $2.23,2.46$, being different from the $g$-values of cytochrome $P-450$ at neutral pH: $1.91,2.22,2.40$ (unpublished results). The results agree with the formation of a new six-coordinated low-spin complex of the heme iron but it cannot be decided if sulfur is retained as one of the axial ligands or is exchanged. 


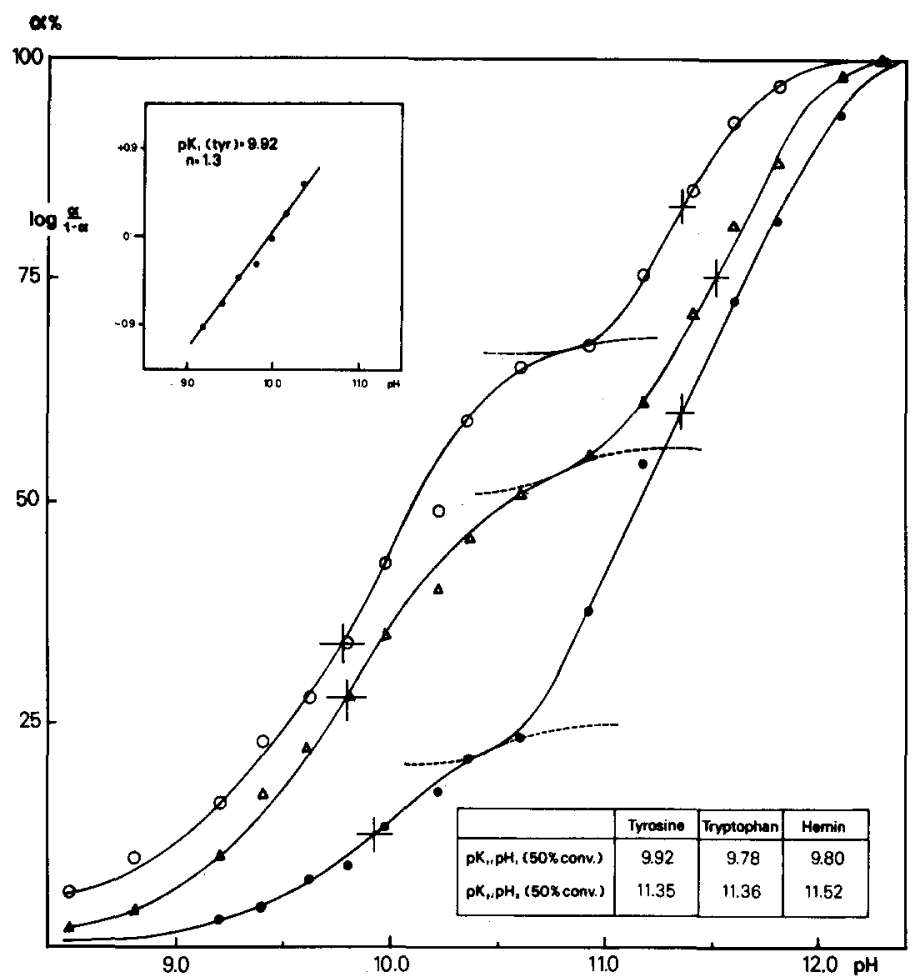

Fig. 6. Dependence of Tyr, Trp and the heme chromophore in cytochrome $P-450$ on pH. From spectral changes in the derivative spectra of Tyr (--) and Trp (- - (Fig. 4) and the difference absorption spectra of the heme chromophore $(O-O)$ (Fig. 5) titration curves were drawn from which the half-conversion points were derived (insert, bottom right). From the Hill plot of the first titration step of Tyr (insert, top left) the $\mathrm{p} K$ as well as the $n$ value were determined.

Further investigations on the structure of the ferric heme complex of cytochrome $P-450$ at alkaline $\mathrm{pH}$ are under study.

In Fig. 6 the $\mathrm{pH}$ dependence of cytochrome $P-450_{\mathrm{LM} 2}$ as reflected in the derivative spectrum of the Tyr and Trp bands as well as the band in the difference absorption spectrum in the Soret region is demonstrated. The $\mathrm{p} K$ of 9.92 , evaluated from the derivative spectrum of the decreasing Tyr band, is almost the same as determined for the ionization of Tyr in the amino acid mixture analogous to cytochrome $P-450_{\mathrm{LM} 2}$. In order to determine whether the first ionization step of Tyr is caused by one or more classes of ionizing Tyr residue, the respective titration values are evaluated according to a Hill plot (see insert in Fig. 6). The resulting value of $n, 1.3$, strongly supports the assumption that only one class of ionizing Tyr is responsible for the first step. The close similarity of the $\mathrm{p} K$ of Tyr in the amino acid mixture to that of Tyr in cytochrome $P-450_{\text {LM2 }}$ indicates that the respective Tyr residue(s) in the protein behaves like that in solution and appears to be exposed on the surface of the molecule and accessible to the solvent.

The titration curve of Tyr in the $\mathrm{pH}$ range from 10.5 to 12.0 shows a second step, from which $\mathrm{p} K_{2}=11.35$ was determined. The decrease of the difference spectrum in the Soret region with increasing $\mathrm{pH}$ also shows two steps; half 
conversion values at $\mathrm{pH} 9.8$ and 11.52 were determined.

Moreover, the two steps observed at each of the other bands (due to Tyr and the heme chromophore in the Soret region) agree with the assumption of two different processes. The second step can be attributed to an unfolding process of the protein, resulting in a similar change of spectral properties of Tyr, Trp and the heme with very similar half transition points. The first step is assumed to be due to the dissociation of Tyr. The similar conversion point of the three bands in the first step suggests that the dissociation of Tyr is somehow linked with Trp and heme.

\section{Discussion}

Following isolation and purification of the essential components (cytochrome $P-450_{\mathrm{LM} 2}$, NADPH-cytochrome $P$-450-reductase and phospholipid) of the liver microsomal monooxygenase system and the demonstration of its reconstitutibility to an enzymatically active system, as reviewed elsewhere [1], recent investigations have been directed to analysis of molecular interactions among these components. For example, it has been shown by Coon and associates [14] that the affinity of cytochrome $P-450_{\mathrm{LM} 2}$ towards substrates is enhanced in the presence of phospholipids and that phospholipid may induce conformational changes in the cytochrome (particularly in cytochrome $\left.P-450_{\mathrm{LM} 4}\right)$ by an increase of the helical content [15] and thus enhance the affinity of reductase for cytochrome $P-450_{\mathrm{LM} 2}$. The formation of a reductasecy tochrome $P-450_{\mathrm{LM} 2}$ complex leads to an increased affinity of cytochrome $P-450_{\mathrm{LM} 2}$ towards substrate, thereby enhancing the high-spin content of the cytochrome. Contrary to the studies with cytochrome $P-450_{\mathrm{LM} 2}$, no conformational changes could be observed by $\mathrm{CD}$ measurements due to interactions between reductase and phospholipid (French, J.S. and Coon, M.J., unpublished results).

Despite such progress, until the present study there have been no data available on the molecular mechanism of these interactions. By means of second derivative difference spectroscopy resolving the $\pi \longrightarrow \pi *$-transitions of the aromatic amino acid residues in the middle ultraviolet region, the contributions of individual amino acid residues to interactions of the components can be analyzed. Participation of such residues in the interactions may be by direct formation of apolar bonds or by indirect conformational changes. Both events, if connected with modification of the environment surrounding the residues, would be reflected in spectral changes which can be determined with high sensitivity using derivative difference spectroscopy.

The interpretation of the results obtained from the interactions between the components has to take into account two effects modifying the derivative spectra of the aromatic amino acids: (i) the polarity of the solvent, and (ii) the degree of ionization of ionizable groups.

(i) Studies on single amino acids and amino acid mixtures corresponding in composition to known proteins with respect to the polarity of the solvent revealed that in 50\% ethanol (v/v) the three amino acids Trp, Tyr and Phe exhibit increased amplitudes of the bands in their derivative spectra amounting to $20-25 \%$. This increase is connected with a red shift of the minima of 
the bands (corresponding to the absorption maxima) [16].

(ii) The $\mathrm{pH}$ titration of Tyr in the amino acid mixture revealed a decrease in amplitude from which a $\mathrm{p} K$ of 9.83 was determined. The Tyr residues of a protein, however, are additionally subjected to a change of the amplitude of their spectra in dependence on the polarity of the solvent. Therefore, the change of the amplitude of the Tyr bands in cytochrome $P-450_{\mathrm{LM} 2}$ represents an overlap between the dissociation of the aromatic hydroxyl group and changed polarity of the solvent due to the unfolding process at alkalization.

The derivative spectra of Tyr in cytochrome $P-450_{\mathrm{LM} 2}$ show 2-2.5-fold higher amplitudes than those in the corresponding amino acid mixture [16]. These higher amplitudes are explicable by the assumption that the residues are localized completely or partially in an apolar environment. Consequently a decrease of the Tyr bands in the derivative spectrum of cytochrome $P-450_{\mathrm{LM} 2}$ with increasing $\mathrm{pH}$ is observed. Such a behavior would be expected upon unfolding of the protein and is also found, indeed, with other hemoproteins such as cytochrome $c$ [16]. The Tyr bands further decrease in amplitude with increasing ionization of the aromatic hydroxyl group. The stepwise decrease in amplitude of the Tyr bands in the range from $\mathrm{pH} 8$ to $\mathrm{pH}>12\left(\mathrm{p} K_{\mathrm{I}}=\right.$ 9.92; $\mathrm{pK}_{2}=11.35$ ), therefore, has to be considered as an overlap of two synergistic effects: the ionization of the hydroxyl group, and the decrease of the hydrophobicity of the environment. Both processes need not be linearly correlated, therefore an estimation of the number of Tyr residues involved in this ionization step is impossible.

The strong similarity between the $\mathrm{p} K_{1}$ of Tyr in cytochrome $P-450_{\text {LM2 }}$ (9.92) and that in the corresponding amino acid mixture (9.83) indicates that the Tyr residues in the protein ionizing with $\mathrm{p} K_{1}$ are exposed to the solvent. Therefore, the observed decrease of the Tyr band is mainly determined by the ionization of the aromatic hydroxyl group and less by environmental changes. The decrease of the Tyr bands in the derivative spectrum of cytochrome $P-450_{\mathrm{LM} 2}$ between $\mathrm{pH} 10$ and $\mathrm{pH}>12$, on the other hand, is caused by the ionization of buried Tyr residues $\left(\mathrm{p} K_{2}=11.35\right)$ which is paralleled by an unfolding process which leads to a decreased hydrophobic environment of the respective Tyr residues.

The interpretation of the striking increase of the Trp amplitude of cytochrome $P-450_{\mathrm{LM} 2}$ at alkaline $\mathrm{pH}$ of the solvent $(\mathrm{pH}>10)$ has to take into account the dependence of the bands at $290 \mathrm{~nm}$ and $283 \mathrm{~nm}$ on the ratio of Trp/Tyr. Balestrieri et al. [5] demonstrated that at Trp/Tyr ratios less than 1 there exists an influence of Tyr on the band at $290 \mathrm{~nm}$, which is properly formed by the Trp absorption. With regard to these interferences and to the ratio $\operatorname{Trp} / \mathrm{Tyr}=1: 9$ in cytochrome $P-450_{\mathrm{LM} 2}$ [10] each change of Trp has to be analyzed carefully before being interpreted in structural terms. Alkalization of the solvent leads to an ionization of the aromatic hydroxyl group of Tyr, which in the derivative spectrum is indicated by a decrease of its amplitude. This decrease of the Tyr amplitude is accompanied by an apparent increase of the Trp amplitude. As the Trp amplitude in cytochrome $P-450_{\mathrm{LM} 2}$ reflects a relatively polar environment, the changes observed are caused by the decrease of the Tyr band inducing concomitant changes in the spectral properties of Trp, rather than by environmental changes of Trp. 
By ESR studies the heme iron in cytochrome $P-450$ has been shown to be preferentially in the low-spin state, indicating a six-coordinated heme iron. Sulfur is generally assumed to be the 5th axial ligand [17]. Different groups, on the other hand, have been proposed as the 6th axial ligand: imidazole [18], $\mathrm{H}_{2} \mathrm{O}$ [19] and the hydroxyl group [20]. The remarkable agreement of the $\mathrm{p} K_{1}$ value of Tyr with the half-conversion point of the decrease of the Soret band leads one to postulate that the hydroxyl group of a Tyr residue is one of the axial heme iron ligands.

It is assumed that the ionization of the postulated heme-bound Tyr is connected with a weakening of the bonds between the iron and the axial ligands. This weakening could be the reason for an exchange of the hemebound sulfur upon the formation of the CO-complex at alkaline $\mathrm{pH}$. Under alkaline conditions this complex shows a Soret absorption at $420 \mathrm{~nm}$ instead of the characteristic position at $450 \mathrm{~nm}$. Obviously in the cytochrome $P-420$ $\mathrm{CO}$ complex the ligand trans to $\mathrm{CO}$ is an imidazole residue as could be derived from infrared studies [21].

Based on the results from the $\mathrm{pH}$-dependence of cytochrome $P-450_{\mathrm{LM} 2}$ the following conclusions are drawn conceming interactions of the essential compounds as observed in the derivative spectra. Upon the interaction of cytochrome $P-450_{\mathrm{LM} 2}$ with phospholipid the Tyr band appears as a minimum in the derivative spectrum, indicating that the amplitude of the Tyr band is enhanced. This increase can be caused by (a) a decrease of the polarity of the immediate environment of the respective residues and/or by (b) a decrease of the degree of ionization of the Tyr residue. The environment of Tyr residues at the surface of the molecule can be directly modified by interaction of these residues with phospholipids. A diminished degree of ionization as another possibility to explain the observed effect is explicable by the assumption that lipid molecules can induce conformational changes leading to a diminished ionization of one or more Tyr residues.

Contrary to the spectral changes induced by phospholipid, the amplitudes of the Tyr band in the derivative spectra decrease upon interaction of cytochrome $P-450_{\text {LM2 }}$ with reductase. In other studies it was found that the interaction of reductase with cytochrome $P-450_{\mathrm{LM} 2}$ shifts the spin equilibrium existing in the heme iron of cytochrome $P-450_{\mathrm{LM} 2}$ [23] to the high-spin state, in the same manner as accomplished by type I substrate (French, J.S. and Coon, M.J., unpublished experiments). This effect of reductase is explicable only by the induction of conformational changes as has been described by $C D$ measurements [15].

Taking into account a $\mathrm{p} K$ of about 10 for the exposed Tyr residue(s) in cytochrome $P-450_{\mathrm{LM} 2}$, it seems very unlikely that the signal in the derivative difference spectrum is caused by the ionization of Tyr, which would be in agreement with a decrease of the Tyr band amplitude. Rather, it has to be assumed that reductase induces conformational changes which by an increase of the polarity in the environment of distinct Tyr residue(s) leads to a decrease of the Tyr bands. From the data so far available it cannot be decided unambiguously which component contributes to the Tyr bands in the difference derivative spectrum of the reductase-cy tochrome $P-450_{\text {LM2 }}$ complex, but the following considerations suggest that the bands originate from cytochrome 
$P-450_{\mathrm{LM} 2}$ rather than from the reductase.

(i) The absence of changes upon interaction of reductase with phospholipids as shown by $\mathrm{CD}$ measurements (French, J.S. and Coon, M.J., unpublished results) as well in the derivative difference spectra, strongly indicates that conformational changes have not been induced and that exposed Tyr residues are not localized at the surface of the reductase molecule.

(ii) The lack of Tyr residues at the surface of the reductase would exclude environmental changes by direct interaction of the reductase with cytochrome $P-450_{\mathrm{LM} 2}$, as well as a shift of the $\mathrm{p} K$ of Tyr residues as necessary prerequisites for a contribution to changes of ionization in the functionally important $\mathrm{pH}$ region.

\section{Acknowledgements}

This research was supported in part by Grant AM-10339 from the United States Public Health Service and Grant PCM 76-14947 from the National Science Foundation to M.J.C. We wish to thank Dr. Schäfer and Dr. Lachman (Institute for Isotopic and Radiation Research, Academy of Sciences of the GDR) for supplying us with dimyristoyl phosphatidylcholine.

\section{References}

1 Coon, M.J., Vermilion, J.L., Vatsis, K.P., French, J.S., Dean, W.L. and Haugen, D.A. (1977) in Drug Metabolism Concepts (Jerina, D.M., ed.), American Chemical Society Symposium Series, No. 44 , 46-71

2 Sandorfy, C. (1961) Die Elektronenspektren in der Organischen Chemie, Verlag Chemie, Weinheim Bergstrasse

3 Ichikawa, T. and Terada, H. (1977) Biochim. Biophys. Acta 494, 267-270

4 Matsushima, A., Inoue, $Y$. and Shibata, K. (1975) Anal. Biochem. 65, 362-368

5 Balestrieri, C., Colonna, G., Giovane, A., Irace, G. and Servillo, L. (1978) Eur. J. Biochem. 90, 433440

6 Hermans, J. (1962) Biochemistry 1, 193-196

7 Leach, S.I. and Scheraga, H.A. (1960) J. Biol. Chem. 2827-2829

8 Wetlau fer, D.B., Edsall, J.T. and Hollingworth, B.R. (1958) J. Biol. Chem. 233, 1421-1428

9 Jung, F., Stopp, G. and Ruckpaul, K. (1965) Nature (London) 207, 990-991

10 Haugen, D.A. and Coon, M.J. (1976) J. Biol. Chem. 251, 7929-7939

11 French, J.S. and Coon, M.J. (1979) Arch. Biochem. Biophys. 195, 565-577

12 Cubero Robles, E. and van den Berg, D. (1969) Biochim. Biophys. Acta 187, 520-526

13 Rauen, H.M. (ed.) (1956) Biochemisches Taschenbuch, Springer Verlag, Berlin

14 Coon, M.J., Haugen, D.A., Guengerich, F.P., Vermilion, J.L. and Dean, W.L. (1976) in The Structural Basis of Membrane Function (Hatefi, Y. and Djavadi-Ohaniance, L., eds.), Academic Press, New York, $409-427$

15 Chiang, Y.-L. and Coon, M.J. (1979) Arch. Biochem. Biophys. 195, 178-187

16 Ruckpaul, K, and Rein, H. (1980) Acta Biol. Med. Germ. 39, 55-69

17 Bayer, E., Hill, H.A.D., Röder, A. and Williams, R.J.P. (1969) Chem. Commun. 109

18 Chevion, M., Peisach, J. and Blumberg, W.E. (1977) J. Biol. Chem. 252, 3637-3645

19 Maricic, S., Vuk-Pavlovic, S., Benko, B., Porok, J., Rein, H., Jänig, G.-R. and Ruckpaul, K. (1977) Croat. Chem. Acta, 49, 323-331

20 Ullich, V., Sakurai, H. and Ruf, H.H. (1979) Acta Biol. Med. Germ. 38, 287-297

21 Rein, H., Böhm, S., Jänig, G.-R. and Ruckpaul, K. (1977) Croat. Chem. Acta 49, $333-338$

22 Sato, R. and Omura, T. (1978) Cytochrome P-450, Kodansha Ltd. Tokyo, Academic Press, New York

23 Rein, H., Ristau, O. Friedrich, U., Jänig, G.-R. and Ruckpaul, K. (1977) FEBS Lett. 75, 19-22 